\title{
Cognitive Function and Self-Care in Patients with Chronic Heart Failure
}

\author{
Jin Shil Kim, PhD1', Seon Young Hwang, PhD², Jae Lan Shim, MS², and Myung Ho Jeong, MD³ \\ 'College of Nursing, Gachon University, Incheon, \\ ${ }^{2}$ College of Nursing, Hanyang University, Seoul, ${ }^{3}$ Department of Cardiology, Chonnam National University, Gwangju, Korea
}

Background and Objectives: This study examined the association of cognitive function with self-care and major adverse cardiac events (MACE) among heart failure (HF) patients.

Subjects and Methods: In this prospective study, 86 outpatients with HF completed face-to-face interviews including neuropsychological testing to evaluate cognitive function and the use of the Self-Care of Heart Failure Index to measure self-care. Functional status was assessed with the New York Heart Association (NYHA) classification. Follow-up data on MACE were obtained at 24 months after enrollment.

Results: Compared with the Korean norm values, more than half of the HF patients had cognitive deficits in global function (33.0\%), immediate recall (65.1\%), delayed recall memory (65.1\%), and executive function (60.5\%). Patients with symptomatic HF ( $\geq$ NYHA class II) had the higher risk for substantially poor cognitive function in all areas of cognitive function than asymptomatic HF patients (NYHA class I, p<0.05). Most patients demonstrated poor self-care adequacy in maintenance (84.9\%), management of symptoms (100\%), and confidence (86.0\%). After adjustment for age and gender, memory function was significantly associated with self-care confidence (odds ratio $1.41,95 \%$ confidence interval $1.03-1.92, p=0.033$ ). No relationship was found between cognition and self-care maintenance. There were 19 MACE's during the 24-month follow-up. Patients without MACE had a significantly higher global cognitive function ( $p=0.024$ ), while no cognitive domains were significant predictors of MACE when adjusted for age and gender.

Conclusion: HF patients with memory loss have poorer self-care confidence. Studies are warranted to examine the functional implication of cognitive deficits and adverse outcomes in a larger sample. (Korean Circ J 2015;45(4):310-316)

KEY WORDS: Heart failure; Cognition; Self care; Outpatients.

\section{Introduction}

Heart failure (HF) is a chronic form of cardiovascular disease (CVD). The prevalence of HF continues to increase, with approximately

Received: October 1, 2014

Revision Received: January 12, 2015

Accepted: March 24, 2015

Correspondence: Seon Young Hwang, PhD, College of Nursing, Hanyang University, 222 Wangsimni-ro, Seongdong-gu, Seoul, 133-791, Korea

Tel: 82-2-2220-0702, Fax: 82-2-2220-0702

E-mail: seon9772@hanyang.ac.kr

- The authors have no financial conflicts of interest.

This is an Open Access article distributed under the terms of the Creative Commons Attribution Non-Commercial License (http://creativecommons. org/licenses/ by-nc/3.0) which permits unrestricted non-commercial use, distribution, and reproduction in any medium, provided the original work is properly cited.
825000 new cases diagnosed each year in the United States. ${ }^{1)}$ In Korea, CVD ranks third in terms of the length of hospitalization and is among the leading diagnoses at discharge. ${ }^{2)}$ The increasing proportion of aged population in Korea may spur the prevalence of $\mathrm{HF}$ associated with fundamental changes in cardiovascular structure and function, which could increase susceptibility to CVD in the senior population.

In HF management, patients with more effective self-care skills exhibit lower mortality and readmission rates than those with poor self-care. ${ }^{4)}$ Self-care is an important strategy in maintaining optimal health for individuals with HF. Given its importance in preventing potentially fatal clinical crises, patient education about self-care behaviors is a key component of HF management in the chronic progression of HF disease.

Cognitive decline may affect a person's decision-making capacity in terms of self-care and interfere with their ability to comply with 
treatment requirements, and to recognize and manage symptom exacerbation. ${ }^{5-7)}$ Cognitive decline in HF often presents deficits in one or more domains of cognition that include attention, memory, executive function, and psychomotor speed. ${ }^{7-9)}$ Cognitive decline or frailty is a particular concern in elderly outpatients with reduced or preserved HF and concomitant comorbidities that complicate early HF diagnosis and management. ${ }^{10111}$ Despite the substantial deficits in one or more areas, few studies have investigated cognitive function in these multiple domains using a comprehensive test battery in Korea. One of the most commonly used neuropsychological tests for cognitive evaluation is the Seoul Neuropsychological Screening Battery. ${ }^{12)}$ The validity and reliability of this comprehensive neuropsychological test battery have been documented from patients with stroke, Parkinson disease, and dementia. ${ }^{12) 13)}$ Empirical evaluation of cognitive function in HF involving global cognition, memory, or executive function, using such a comprehensive neuropsychological test battery is lacking.

Cognitive decline adversely affects adherence to therapeutic regimens for HF management and major adverse cardiac events (MACE) in western countries. ${ }^{5 / 8) 14-16)}$ However, little is known about the relationship between cognitive decline and self-care among HF patients in Korea.

The present study had four purposes: to estimate the levels of cognitive function in domains of global, memory, and executive function; examine differences in each domain of cognitive function between patients with asymptomatic and symptomatic HF; examine the association of cognitive function with self-care adherence in Korean HF patients; and examine the influence of cognitive impact on MACE. The study involved Korean HF patients with preserved and reduced left ventricular ejection fraction (LVEF).

\section{Subjects and Methods}

\section{Study design and subjects}

This study adopted a prospective study design in which associations of cognitive function with self-care adherence and MACE at 24 months after the enrollment were examined in HF patients. Study participants were recruited and enrolled from a national university-affiliated outpatient clinic in 2012. Eligible criteria included adult patients who were over 30 years of age, HF diagnosed at least 6 months previously, ability to communicate verbally, and voluntary informed consent to participate. Patients with a documented history of disorders potentially associated with cognitive deficits, such as stroke, dementia or Alzheimer's disease, were excluded. The relevant institutional review board approved the study protocol (CHUH-2012-091). Written informed consent statement was obtained from each participant before a face-to- face interview for neuropsychological tests to evaluate cognition, self-care, and functional classification.

\section{Measurements and data collection}

\section{Cognitive function}

Cognitive function was measured with the Seoul Neuropsychological Screening Battery ${ }^{12)}$ which was established to assess comprehensive neuropsychological aspects involving global cognition (Korean-Mini Mental State Examination, K-MMSE), memory (Seoul Verbal Learning Test), and executive function (Controlled Oral Word Association).

\section{Global cognition}

The Mini Mental State Examination (MMSE) is a measure of global cognition originally developed by Folstein et al. ${ }^{17)}$ The K-MMSE is a standardized and modified form of the MMSE for elderly Korean people. The K-MMSE is a 30 item-instrument with possible scores ranging from 0 to 30, with higher scores indicating greater general cognition. $^{18)}$

\section{Memory}

Memory function contains both immediate and delayed recall memory. It was measured with the Seoul Verbal Learning Test that involves oral presentation using a list of 12 words to the patients during three trials. Patients were asked to recall and repeat the words in any order after each trial (total list learning, immediate recall, range 0-36). Twenty minutes later, when the third trial was complete, patients were asked to recall as many of the 12 words they could (delayed recall, range $0-12)^{12)}$

\section{Executive function}

Executive function is an umbrella term for the regulation or control of cognitive processes including working memory, reasoning, and problem solving as well as planning and execution. ${ }^{19)}$ It was measured using the Controlled Oral Word Association test by presenting three letters to the patients. The patients were asked to generate as many words beginning with each letter as they could over a 60-s period. The number of words generated for the three letters indicated an individual score of executive function, with higher scores indicating greater function. ${ }^{12)}$

\section{Self-care}

Self-care is defined as a naturalistic decision-making process involving the choice of behaviors that maintain physiologic stability (maintenance) and the response to symptoms when they occur (management), and the confidence in self-care for HF management. ${ }^{20)}$ Self-care was measured with the Self-Care of Heart 
Failure Index ${ }^{211}$ which included three subscale scores associated with self-care maintenance (10 items; behavioral adherence to some of the major treatment recommendations such as checking weight and ankle swelling, exercise, taking medication, keeping physician's appointment, and low-sodium diet), self-care management (6 items; when dyspnea or leg edema occur, ability to recognize symptom changes, implement remedies, and evaluate their effectiveness), and self-care confidence ( 6 items; confidence to engage in each phase of the self-care process). Self-care management was assessed only for patients who indicated having experienced dyspnea or a leg edema. Scores of maintenance and management reflect the degrees of self-care ability, and confidence scores explain how some patients master self-care and others do not. ${ }^{211}$ Responses from each of the three self-care subscales were transformed to 100 points; higher scores reflected superior self-care. Self-care adequacy for each subscale area was determined using a cut-off point $\geq 70$. ${ }^{\text {.20) }}$

\section{Functional classes}

New York Heart Association (NYHA) functional classification was used in this study, as it is the most widely-used system to categorize patients according to the degrees of functional limitation imposed by HF symptom severity. ${ }^{22)}$ Patient data on cognitive function, selfcare, and functional classes were collected by a trained research nurse from February to September 2012 through a 60-minute, oneon-one interview. Follow-up clinical data including MACE were obtained 24 months after the enrollment by the research nurse.

\section{Statistical analyses}

Data were analyzed using SPSS version 18.0 (SPSS Inc., Chicago, IL, USA). Descriptive statistics were completed for all study variables, including frequency. Data are presented as meantstandard deviation, with ranges for continuous variables and percentages for categorical variables. Neuropsychological test variables were scored following author guidelines, ${ }^{12)}$ with raw scores for general cognition, memory, and executive function. Student's t-tests were performed to examine differences in cognitive function and self-care between patients with asymptomatic and symptomatic HF. A series of multiple logistic regression analyses were also performed to determine factors that predicted self-care and MACE in HF patients. For logistic regression analyses, the subscales of self-care maintenance and confidence were dichotomized for determination of self-care adequacy using a cut-off point of 70 .

\section{Results}

The sociodemographic and clinical characteristics of the sample are presented in Table 1. Eighty six HF patients completed the face-to-face
Table 1. Characteristics of the 86 subjects

\begin{tabular}{|c|c|}
\hline Variables & Values \\
\hline Age (years) & $58.3 \pm 12.9$ \\
\hline $30-60$ & $43(50.0)$ \\
\hline $61-79$ & $43(50.0)$ \\
\hline \multicolumn{2}{|l|}{ Gender } \\
\hline Male & $58(67.4)$ \\
\hline \multicolumn{2}{|l|}{ Marital status } \\
\hline Unmarried, widowed, divorced & $24(27.9)$ \\
\hline \multicolumn{2}{|l|}{ Education } \\
\hline$\leq$ Elementary school & $21(25.5)$ \\
\hline Middle-high school & $46(53.5)$ \\
\hline$\geq$ College & $19(22.0)$ \\
\hline \multicolumn{2}{|l|}{ Occupation } \\
\hline Yes & $40(46.5)$ \\
\hline \multicolumn{2}{|l|}{ Etiology } \\
\hline Ischemic & $18(20.9)$ \\
\hline Idiopathic & $5(5.8)$ \\
\hline Hypertension & $13(15.1)$ \\
\hline Valvular & $14(17.5)$ \\
\hline Cardiomyopathy/others & $36(41.7)$ \\
\hline HF duration (months) & $38.2 \pm 34.5$ \\
\hline $6-24$ & $45(55.0)$ \\
\hline $25-159$ & $41(45.0)$ \\
\hline Frequency of hospitalization & $1.5 \pm 1.6$ \\
\hline Systolic blood pressure (mmHg) & $126.7 \pm 21.1$ \\
\hline Diastolic blood pressure $(\mathrm{mmHg})$ & $78.6 \pm 14.9$ \\
\hline LVEF \% & $51.2 \pm 14.6$ \\
\hline $45 \%$ & $19(22.1)$ \\
\hline \multicolumn{2}{|l|}{ NYHA class } \\
\hline$\|$ & $35(40.7)$ \\
\hline III \& IV & $8(9.3)$ \\
\hline \multicolumn{2}{|l|}{ MACEs } \\
\hline Cardiac death & $2(2.3)$ \\
\hline Readmission & 17 (19.8) \\
\hline Infarction & $3(3.5)$ \\
\hline Cardiomyopathy & $3(3.5)$ \\
\hline Angina, dyspnea, dizziness & $11(12.8)$ \\
\hline Current smokers & 16 (18.6) \\
\hline Diabetes & $13(15.1)$ \\
\hline Atrial fibrillation & $15(17.4)$ \\
\hline \multicolumn{2}{|l|}{ Medications } \\
\hline$\beta$-blocker & $68(79.1)$ \\
\hline ACE-inhibitor & $21(24.4)$ \\
\hline Angiotensin receptor blocker & $53(61.6)$ \\
\hline Diuretics & $56(65.1)$ \\
\hline Statin & $44(51.2)$ \\
\hline
\end{tabular}

Values are mean \pm standard deviation or number (\%). HF: heart failure, LVEF: left ventricular ejection fraction, NYHA: New York Heart Association, MACEs: major adverse cardiac events, ACE: angiotensin converting enzyme 
interviews. The mean age of the patients was $58.32 \pm 12.87$ years and $67.4 \%(n=58)$ were men. Seventy percent of patients were married and $30 \%$ lived alone. More than half of the patients (55\%) had been diagnosed with HF for 24 months or longer before enrollment. Half of the patients were asymptomatic (NYHA class I). The mean value of the LVEF was $52.15 \pm 14.61 \%$ (range 20.2-75.0). Approximately, one in five patients $(n=19,22 \%)$ had reduced LVEF $<45 \%$. During the 24-month follow-up there were 67 event-free survivors; 19 MACE's including two deaths occurred. HF patients were on optimal medication including angiotensin converting enzyme inhibitors, angiotensin receptor blockers, beta-blockers, and diuretics.

Cognitive function and self-care level data are presented in Tables 2 and 3. Using the Korean norm values for comparisons, 33.0\%, $65.1 \%$, and $65.1 \%$, and $60.5 \%$ of HF patients had a cognitive deficit in global cognition, immediate and delayed memory, and executive function, respectively. Using a score of 70 as the cut-off to determine self-care adequacy for each subscale, $84.9 \%$ and $86.0 \%$ patients showed inadequate self-care maintenance and confidence, respectively. All patients who had dyspnea or leg edema $(n=35)$ had inadequate self-care management with scores below the norm. All subscale scores of self-care were significantly lower in symptomatic HF patients ( $\geq$ NYHA class II) than in asymptomatic HF patients (NYHA class I; $p<0.05)$. Compared with asymptomatic HF patients, symptomatic HF patients also had poorer cognitive function in domains of general, executive function, and memory $(p<0.05)$ (Table 4). However, no significant differences were found in cognitive function and self-care between HF patients with preserved and reduced LVEF ( $p>0.05)$.

Multivariate logistic regression analyses indicated that delayed recall memory \{odds ratio (OR) 1.41, 95\% confidence interval (CI) $1.03-1.92, p=0.033\}$ was an independent predicting factor of selfcare confidence adequacy after controlling for age and gender. There was no significant relationship between cognitive function and self-care maintenance in the multiple logistic regression analysis (Table 5). In comparisons of cognitive function between

Table 2. Neuropsychological test scores in general cognitive function

\begin{tabular}{lcccc}
\hline Measure & Raw score & Range & Norm & <Norm \\
\hline Global function (K-MMSE) & $26.4 \pm 5.3$ & $14-30$ & $26.8 \pm 3.3$ & $28(33.0)$ \\
Memory function (SVLT) & & & $17.6 \pm 4.5$ & $56(65.1)$ \\
$\quad$ Immediate recall & $15.5 \pm 5.8$ & $2-31$ & $5.7 \pm 2.4$ & $56(65.1)$ \\
$\quad$ Delayed recall & $4.8 \pm 2.3$ & $0-9$ & $22.9 \pm 9.3$ & $52(60.5)$ \\
\hline Executive function (COWA) & $20.1 \pm 10.2$ & $0-51$ & \\
\hline
\end{tabular}

Values are mean \pm standard deviation or number (\%). K-MMSE: Korean mini-mental state examination, SVLT: Seoul verbal learning test, COWA: controlled oral word association

Table 3. Descriptive statistics for self-care of the subjects

\begin{tabular}{lrrr}
\hline & Range & M \pm SD & Poor <70* \\
\hline Self-care maintenance $(n=86)$ & $20.0-89.9$ & $55.4 \pm 14.3$ & $73(84.9)$ \\
Self-care management ${ }^{+}(n=35)$ & $5.0-60.0$ & $34.0 \pm 12.8$ & $35(100.0)$ \\
Self-care confidence $(n=86)$ & $5.5-94.5$ & $52.1 \pm 17.6$ & $74(86.0)$ \\
\hline
\end{tabular}

* Values are expressed as numbers (\%). ${ }^{+}$Assessed only for patients who had dyspnea or leg edema

Table 4. Self-care and cognitive function between patients with asymptomatic and symptomatic heart failure

\begin{tabular}{|c|c|c|c|c|}
\hline & $\begin{array}{l}\text { Asymptomatic NYHA I } \\
\qquad(n=43)\end{array}$ & $\begin{array}{l}\text { Symptomatic NYHA } \geq 1 \mathrm{I} \\
\qquad(n=35)\end{array}$ & $t$ & $p$ \\
\hline Self-care maintenance & $57.4 \pm 14.6$ & $53.4 \pm 13.8$ & 1.33 & 0.185 \\
\hline Global function (K-MMSE) & $27.8 \pm 2.5$ & $24.9 \pm 4.4$ & 3.62 & 0.001 \\
\hline \multicolumn{5}{|l|}{ Memory function (SVLT) } \\
\hline Executive function (COWA) & $23.4 \pm 9.8$ & $16.9 \pm 9.6$ & 3.14 & 0.002 \\
\hline
\end{tabular}

NYHA: New York Heart Association, K-MMSE: Korean mini-mental state examination, SVLT: Seoul verbal learning test, COWA: controlled oral word association 
Table 5. Factors predicting self-care confidence by multiple logistic regression analysis, adjusted for age and gender

\begin{tabular}{|c|c|c|c|c|c|}
\hline Variables & B & SE & $\operatorname{Exp}(B)$ & $95 \% \mathrm{Cl}$ & $p$ \\
\hline Global function & 0.02 & 0.10 & 0.92 & $0.83-1.26$ & 0.850 \\
\hline Delayed recall memory & 0.87 & 0.30 & 1.41 & $1.03-1.92$ & 0.033 \\
\hline Executive function & 0.04 & 0.04 & 0.99 & $0.91-1.08$ & 0.995 \\
\hline NYHA class & 0.28 & 0.72 & 1.33 & $0.32-5.47$ & 0.698 \\
\hline HF duration & -0.01 & 0.01 & 0.99 & $0.97-1.00$ & 0.303 \\
\hline
\end{tabular}

B: Beta, SE: standard error, Exp: exponential, Cl: confidence interval, NYHA: New York Heart Association, HF: heart failure

event-free survivors and those with MACE, event-free survival was associated significantly with higher global cognitive function (K-MMSE; 23.9 vs. 27.1; $t=2.30 \mathrm{p}=0.024$ ). Other areas of cognitive function did not significantly differ between the two groups: immediate recall ( 14.5 vs. $15.6, t=0.74 p=0.462$ ), delayed recall memory (4.7 vs. $4.9, t=0.33 p=0.773$ ), and executive function (16.9 vs. 21.0, $t=1.17 p=0.085)$. However, multiple logistic regression analysis showed that, for testing cognitive impacts on the likelihood of MACE, none of the cognitive domains were significant predictors of MACEs after adjusting for age, gender, and LVEF.

\section{Discussion}

This is the only study to our knowledge that has investigated cognitive function in HF using a neuropsychological test battery. ${ }^{12)} \mathrm{A}$ major finding was that substantial extent of poor cognitive function in Korean HF patients, with the deficits poorer among patients who had more functional limitation indicated by NYHA class II and higher than those with NYHA class I. Such cognitive deficits adversely affected self-care in Korean HF patients. In particular, delayed recall memory was predictive for poor self-care confidence adequacy.

Poor cognitive function was evident in all domains of global, immediate, and delayed recall memory, and executive function, with the prevalence of cognitive deficit ranging from $33.0 \%$ to $63.8 \%$, compared with the Korean norm values of neuropsychological test scores. ${ }^{12)}$ Post hoc analysis was performed to examine whether cognitive dysfunction differed by preserved and reduced LVEF. Compared with HF patients with preserved LVEF, there were no differences in all domains of cognitive function among HF patients with low LVEF. These results may reflect the small sample size. Poor cognitive function is well-documented in HF; chronic cerebral hypoperfusion and, subsequently, ischemic structural change and functional alteration are mostly responsible for the impairment. ${ }^{9)^{16) 23)}}$ Particularly, adults with HF showed an evidence of cerebral grey matter loss, and had worse immediate and long-term memory and psychomotor speed than controls without ischemic heart disease. ${ }^{24)}$ Further research is needed to determine the differences of cognitive function by preserved and reduced LVEF in a larger sample.

Our findings are consistent with a study that investigated cognitive function among $121 \mathrm{HF}$ patients in Korea. ${ }^{25)}$ Using a single screening measure of the K-MMSE to examine cognitive function and a score $<20$ as a cut-off, 29 patients (24\%) had a cognitive deficit. $^{25)}$ Pressler et al. ${ }^{23)}$ also found that global cognitive function (MMSE, $p=0.004$ ), working memory (digit span backward, $p=0.018$ ), and memory (Hopkins Verbal Learning Test delayed recall, $p=0.047$ ) were poorer among $166 \mathrm{HF}$ patients with left ventricular dysfunction, compared with those having chronic illness other than HF or healthy people. A systematic literature review also indicated that patients with severe HF can have mild cognitive deficit, with the prevalence of cognitive deficit being $53-58 \%$ of patients. ${ }^{16)}$

When comparing cognitive function by functional limitation imposed by HF symptoms, patients with symptomatic HF ( $\geq N Y H A$ class II) had substantially poorer cognitive function in all domains than patients with asymptomatic HF (NYHA class I; $p<0.05$ ). This finding validates a previous report that pilot-tested cognitive function among seven HF patients. ${ }^{22)}$ In this pilot test, poor global cognition, memory, and executive function were found in more than half of patients, with 57\%,50\%, and $83 \%$ having poor global cognition, memory, and executive function, respectively. In another study, NYHA class I or II had greater cognitive function than those in NYHA class III and higher. ${ }^{26)}$

Most patients with HF in this study demonstrated poor self-care adequacy in maintenance (84.9\%), management (100\%), and confidence (86.0\%), using the cut-off score of 70. Especially, all 35 patients who acknowledged dyspnea or leg edema did not response properly to symptoms when they occurred. Self-care management is an active, deliberate process that recognizes a change in health, evaluating the change, deciding to take action, and implementing a treatment strategy. ${ }^{20)}$ Symptom monitoring is an essential segue into self-care management, which refers to decision-making in response to symptoms. ${ }^{20)}$ An educational strategy is valuable to help patients monitor their symptoms regularly and recognize early worsening symptom(s) on a daily basis.

Those who were symptomatic and had more functional limitation, 
as indicated by NYHA class II or higher, also had poorer self-care confidence compared with those who were asymptomatic (NYHA class I). In a previous pilot study, poor self-care maintenance and management were observed; cognitive function was substantially poorer in Korean HF patients who showed inadequate self-care adherence. ${ }^{26)}$ Our findings were consistent with this pilot report, further validating poor self-care in a larger sample of Korean HF patients. More important and consistent with results from previous studies, delayed recall memory was a significant factor that may have affected self-care confidence in HF patients in this study.

However, cognitive function between event-free survivors and those with MACE was not significantly different, except for global function, with event-free survival having a higher global function. Multiple logistic regression analyses showed that no cognitive domain predicted MACE among HF patients with both preserved and reduced LVEF. On the contrary, data from a larger sample of HF patients with low LVEF ( $n=166,27.0 \pm 10.1)$ support that cognitive deficit is associated with adverse health outcomes. ${ }^{23)}$ Poor cognitive function in the domains of global cognition, working memory, psychomotor speed, and executive function were reported to be significant predictors of 12-month all-cause mortality in a series of logistic regression analyses with LVEF, with each cognitive function variable as a predictor. ${ }^{23)}$ Although memory loss explained only $1 \%$ of the variance, HF severity, age, depressive symptoms, and immediate recall memory were significant predictors of healthrelated quality of life in HF patients $(n=249) .{ }^{27)}$ Given that damaged areas associated with cerebral hypoxia may prevent HF patients from engaging in self-care activities for HF management, ${ }^{23)}$ our results add empirical evidence in Korean $\mathrm{HF}$ research, with outcome measures expanding to daily functional ability, self-care, and MACE on cognitive deficits in $\mathrm{HF}$ involving those with preserved or reduced LVEF; and cognitive function as a critical modifiable factor with regard to improving such health outcomes.

\section{Limitations}

There were two limitations to this study. First, the findings should be validated in comparison with other patient groups and healthy individuals using a larger sample. Second, patients who participated in this study were recruited from a single universityaffiliated hospital, limiting the generalization of the results.

\section{Conclusion}

Korean HF patients showed poor cognitive function and inadequate levels of self-care maintenance, management, and confidence. Those with more functional limitation demonstrated poorer cognitive function and worse self-care confidence than their counterparts. Importantly, memory loss was a significant predictor of self-care confidence. Future research is warranted to determine how cognitive deficits impede self-care decision-making processes separately in preserved and reduced HF patients over a longer time frame. Further, intervention studies are needed to learn how to delay or prevent poor cognitive function in HF patients.

\section{Acknowledgments}

This study was supported by a grant from the Korean Society of Cardiology (2012).

\section{References}

1. Go AS, Mozaffarian D, Roger VL, et al. Heart disease and stroke statistics--2014 update: a report from the American Heart Association. Circulation 2014;129: e28-e292.

2. Health Insurance Review \&t Assessment Service. Chronic disease summary statistics in South Korea. Available from: http://www.hira. or.kr/rd/dissdic/hifreqdiselnfo.do?pgmid=HIRAA020044020400.

3. Chen J, Dharmarajan K, Wang Y, Krumholz HM. National trends in heart failure hospital stay rates, 2001 to 2009. J Am Coll Cardiol 2013;61:1078-88.

4. Lee CS, Moser DK, Lennie TA, Riegel B. Event-free survival in adults with heart failure who engage in self-care management. Heart Lung 2011;40:12-20.

5. Rigel B, Moser D, Anker SD, et al. State of the science: promoting selfcare in persons with heart failure: a scientific statement from the American Heart Association. Circulation 2009;120:1141-63.

6. Eggermont LH, de Boer K, Muller M, Jaschke AC, Kamp O, Scherder EJ. Cardiac disease and cognitive impairment: a systematic review. Heart 2012;98:1334-40.

7. Riegel $B$, Lee CS, Dickson W. Self care in patients with chronic heart failure. Nat Rev Cardio/ 2011;8:644-54.

8. Pressler SJ, Subramanian U, Kareken D, et al. Cognitive deficits in chronic heart failure. Nurs Res 2010;59:127-39.

9. Dardiotis E, Giamouzis G, Mastrogiannis D, et al. Cognitive impairment in heart failure. Cardiol Res Pract 2012;595821.

10. Heckman GA, Demers C, Hogan DB, McKelvie, RS. Heart failure: old disease, older adults, fresh perspective. Geriatr Aging 2008;11:15-21.

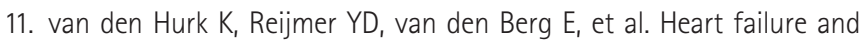
cognitive function in the general population: the Hoorn Study. Eur $J$ Heart Fail 2011;13:1362-9.

12. Kang Y, Na DL. Seoul Neuropsychological Screening Battery (SNSB). Seoul: Human Brain Research \& Consulting Co; 2003.

13. Ahn HJ, Chin J, Park A, et al. Seoul neuropsychological screening battery-dementia version (SNSB-D): a useful tool for assessing and monitoring cognitive impairments in dementia patients. J Korean Med Sci2010;25:1071-6.

14. Zuccalà G, Onder G, Pedone $C$, et al. Cognitive dysfunction as a major determinant of disability in patients with heart failure: results from a multicentre survey. On behalf of the GIFA (SIGG-ONLUS) Investigators. J Neurol Neurosurg Psychiatry 2001;70:109-12.

15. Cameron J, Worrall-Carter L, Riegel B, Lo SK, Stewart S. Testing a model 
of patient characteristics, psychologic status, and cognitive function as predictors of self-care in persons with chronic heart failure. Heart Lung 2009;38:410-8.

16. Vogels RL, Scheltens $P$, Schroeder-Tanka JM, Weinstein HC. Cognitive impairment in heart failure: a systematic review of the literature. Eur J Heart Fail 2007:9:440-9.

17. Folstein MF, Folstein SE, McHugh PR. "Mini-mental state". A practical method for grading the cognitive state of patients for the clinician. $J$ Psychiatr Res 1975;12:189-98.

18. Kang $Y$, Na DL, Hahn S. A validity study on the Korean mini-mental state examination (K-MMSE) in dementia patients. J Korean Neurol Assoc 1997;15:300-8.

19. Elliott R. Executive functions and their disorders. Br Med Bull 2003;65:49-59.

20. Riegel B, Lee CS, Dickson W, Carlson B. An update on the self-care of heart failure index. J Cardiovasc Nurs 2009;24:485-97.

21. Riegel $B$, Dickson W. A situation-specific theory of heart failure selfcare. J Cardiovasc Nurs 2008;23:190-6.

22. Hunt SA, Abraham WT, Chin MH, et al. ACC/AHA 2005 guideline update for the diagnosis and management of chronic heart failure in the adult: a report of the American College of Cardiology/American Heart Association Task Force on practice guidelines (Writing Committee to update the 2001 guidelines for the evaluation and management of heart failure): developed in collaboration with the American College of Chest Physicians and the International Society for Heart and Lung Transplantation: endorsed by the Heart Rhythm Society. Circulation 2005;112:e154-235.

23. Pressler SJ, Kim J, Riley P, Ronis DL, Gradus-Pizlo I. Memory dysfunction, psychomotor slowing, and decreased executive function predict mortality in patients with heart failure and low ejection fraction. J Card Fail 2010;16:750-60.

24. Almeida OP, Garrido GJ, Beer C, Lautenschlager NT, Arnolda L, Flicker L. Cognitive and brain changes associated with ischemic heart disease and heart failure. Eur Heart J 2012;33:1769-76.

25. Son YJ, Kim SH, Kim GY. Factors influencing adherence to self care in patients with chronic heart failure. Korean J Adult Nurs 2011;23:24454.

26. Kim JS, Shin JH. Cognitive function and self-care in patients with heart failure: a pilot study from Korean patients. Perspect Nurs Sci 2012;9:45-50.

27. Pressler SJ, Subramanian U, Kareken D, et al. Cognitive deficits and health-related quality of life in chronic heart failure. $J$ Cardiovasc Nurs 2010;25:189-98. 